Pieric Ferrari, Kai Nagel

\title{
Robustness of Efficient Passenger Boarding Strategies for Airplanes
}

Journal article | Accepted manuscript (Postprint)

This version is available at https://doi.org/10.14279/depositonce-7800

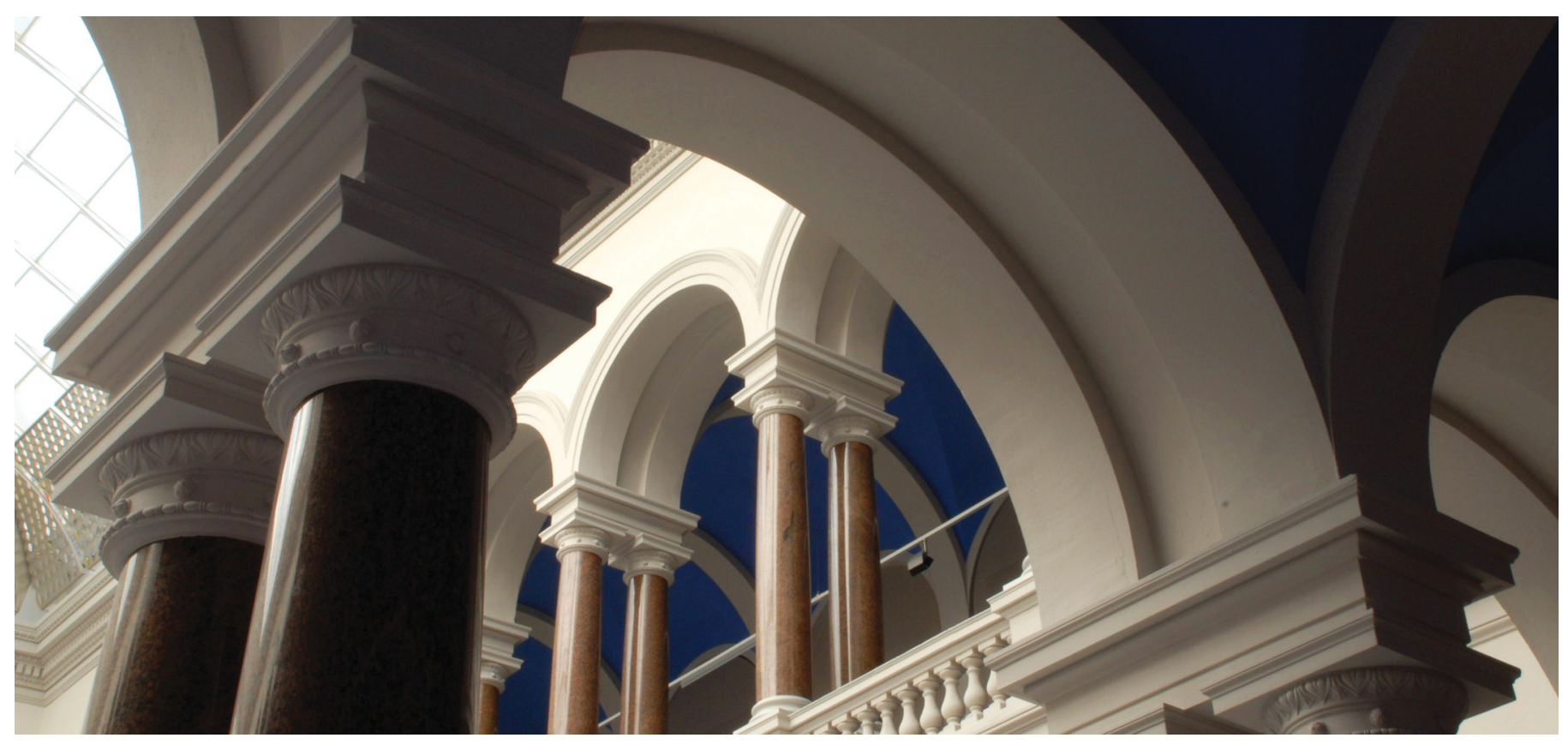

Pieric Ferrari, Kai Nagel, Robustness of Efficient Passenger Boarding Strategies for Airplanes,

Transportation Research Record: Journal of the Transportation Research Board (Vol 1915) pp. 44-54.

Copyright (c) [2005] (Sage). DOI: [10.3141/1915-06]. 


\title{
Robustness of Efficient Passenger Boarding Strategies for Airplanes
}

\author{
Pieric Ferrari and Kai Nagel
}

\begin{abstract}
Common wisdom is that airplanes make money only when they are in the air. Therefore, turnaround time (turn time) on the ground should be reduced as much as possible. An important contribution to the turn time is airplane boarding time. Many different schemes are in use, from random seat selection to sophisticated boarding groups. A simulation model is described to evaluate different boarding strategies. In contrast to earlier work, it puts special emphasis on disturbances, such as a certain number of passengers not following their boarding group but boarding earlier or later. A surprising result of this work is that the typical back-to-front boarding strategy becomes improved when passengers do not board with their assigned group. Other proposed strategies still consist of small numbers of boarding groups but are both faster and more robust with regard to disturbances.
\end{abstract}

Today's airline problems are based on a competitive atmosphere and pricing pressure. Companies have to reduce their costs at every level. Airplanes have to work at full capacity, and airplane turnaround time (turn time) has to be minimized. Nevertheless, punctuality of flights should be ensured. Large groups of passengers waiting for hours and overcrowded airport facilities indicate that this goal is not yet achievable in the holiday season. To reduce the idle time of airplanes, optimizations can start at any point between arrival and departure.

Turn time depends on the deplaning process, aircraft cleaning, and passenger boarding. Passenger boarding is the element that takes the longest time and is therefore the most important. Different solutions have been proposed to reduce boarding time, and many airlines use different methods. The main goal is to get passengers into their seats in a fast and efficient way. This process is highly dependent on passenger behavior inside the aircraft. The aisle is typically narrow, passengers are often carrying luggage that prevents them from passing through, and even a few persons can delay the whole process.

To reduce disturbances, passengers are often divided into boarding groups that enter the aircraft one after another. Inside the aircraft these groups are arranged among the seats in a manner that should avoid conflicts between passengers. The number of boarding groups should be as small as possible, otherwise their announcement becomes com-plicated. To test the behavior of boarding strategies under different conditions, a sophisticated simulation environment was designed. It not only enables a closer look at existing solutions but also allows the easy implementation of new strategies to achieve even better results.

P. Ferrari, Department of Computer Science, Swiss Federal Institute of Technology, ETH Zürich, Beckenhofstrasse CH-64, Zürich 8006, Switzerland. K. Nagel, Institute for Land and Sea Transport Systems, Salzafer 17-19, Building 12, Technische Universität, Berlin 10587, Germany.
The solutions proposed in this paper will improve the passenger boarding process considerably. Airlines introducing such strategies will reduce their costs by minimizing the turn time of their fleet.

\section{EARLIER SOLUTIONS}

Only a few published papers were found on optimizing the passenger boarding process for airplanes. A wide palette of boarding strategies was simulated systematically by Van Landeghem and Beuselinck (VLB) (1). Their results will be discussed throughout the paper and compared with the results of the current study.

The aircraft boarding problem was also analyzed theoretically as a nonlinear assignment problem (2). The problem was modeled as a binary integer program in which the objective function was the minimization of the total number of interferences. The study showed that outside-in loading strategies perform better than back-to-front strategies do. Disturbances were not taken into account.

The whole turn time process was investigated by using an enplanedeplane simulation for Boeing (3). Various interior configurations of a Boeing 757 were tested with different boarding strategies by using a discrete event simulation. Boeing also verified the results with real passengers representing a typical traveling population. Kirchner at al. used a simulation technique similar to the one used in the current study (4). They concentrate, however, on egress behavior, which is easier to model since all passengers have the same destination (outside). It is easier even if there are multiple exit doors, since even with multiple exit doors, the driving force can be generated by a single potential that is the same for all passengers. In contrast to other studies, Kirchner et al. compared their simulation results with actual field measurements. In consequence, a fair amount of effort in their work was spent on calibration and sensitivity testing with respect to passenger movement parameters.

A combinatorial boarding model that uses space-time and Lorentzian geometry was proposed by Bachmat et al. (5). The diameter of space-time then provides the expected boarding time. They studied how the airplane interior design (leg room) affects boarding and reproduced some of the strategies tested by VLB. There seems to be good agreement with VLB's work when few boarding groups are used.

\section{CURRENT SOLUTION}

The current examination is based on the studies by VLB. This work differs from their work in some important aspects:

- Whereas their model uses random process times with triangular distribution for passenger movement, the current model applies deterministic constant process times as described in the following section 
on the passenger model. In addition, some details such as luggage loading delay are not fully described by VLB. It turns out that the results are robust under such simplifications and assumptions. The main result is, as in the studies by VLB, that completely random boarding is faster than the standard back-to-front boarding used by many airlines.

- The current study also looks at the robustness of the strategies under disturbances. Disturbance means that passengers do not enter with their assigned boarding groups but earlier or later. Since early boarding can be prevented by the airline staff, early boarding is treated separately from late boarding. Then this study also looks at strategies that are both simple to implement and robust under such disturbances. Rather obviously, after the result mentioned earlier-that random boarding is faster than back-to-front boarding - the typical back-tofront boarding strategy becomes more efficient under disturbances. That is, in the interest of efficient boarding, passengers should be encouraged not to follow the row announcements. There are, however, strategies that are more efficient than random boarding, and predictably, their performance degrades under disturbances.

\section{Aircraft Model}

For this study, optimization of short-haul flights is of interest because the percentage of turn time for such flights is much greater than is that for long intercontinental flights. For comparability, the same standard airplane is used as that used by VLB, which is typical for such flights. The aircraft has 132 seats in 23 rows. Rows 1 and 23 have three seats only; the others have six. It is clear that the same simulation model can be used for other airplanes.

An entirely cell-based representation of the airplane is used: the plane is discretized into rectangles, in which every seat and the width of the aisle correspond to exactly one field. It is assumed that a passenger with luggage takes as much room as a seat, and no space is modeled between the rows. The airplane is always entered by the front door. One important result of this study will be that such a simplified representation of space leads to results that are very similar to the results obtained with a model with continuous space representation.

\section{Passenger Model}

VLB use a triangular distribution for passenger movement, with the values 1.8, 2.4, 3 (i.e., a triangle starting at 1.8, reaching its maximum at 2.4, and ending at 3). The model used in this study, in contrast, uses a deterministic process, in which a passenger can move one cell or row forward per time step if the destination cell is free. This process means that one time step of the simulation corresponds to $2.4 \mathrm{~s}$ of VLB's simulation. Only the efficiency of strategies measured in steps will be compared, and absolute boarding times will not be calculated.

During one simulation time step all cells representing passengers are processed once and in random order. All actions are based on one grid; therefore the simulation implements a serial update of passengers' status and position.

Passengers enter the front door and queue in a single line until they reach their assigned seats. They then put their carry-on luggage into the overhead bin or underneath the seat and sit down. Different conflicts can occur during this process:

- As passengers enter, the overhead bin fills up and it takes longer to find free room for luggage. They may even have to move to another row to store their luggage, but this will not be included in the simulation.

- A passenger seated in an aisle seat is in the way if another passenger has to get to the window seat. In this case the sitting passenger has to get up, leave the row, and sit down again after the passenger sitting next to the window has sat down. This kind of interference is called seat interference.

In both cases, upstream passengers need to wait until the process is finished.

\section{Bin Occupancy Model}

There is an overhead bin for each row on each side of the aisle. A bin occupancy model is used that is similar to that used in the simulation by VLB. To every passenger a random number of pieces of luggage is assigned by using the following distribution: $60 \%$ of the passengers are carrying one piece of luggage, $30 \%$ have two pieces of luggage, and $10 \%$ of them have three pieces of luggage.

The time (in simulation time steps) that the travelers need to store their pieces of luggage depends on the luggage they carry and the occupancy of the overhead bin:

$t_{s l}=2+\frac{n_{\mathrm{bin}}+n_{l}}{2} * n_{l}$

where

$t_{s l}=$ time to store all pieces of luggage (simulation time steps),

$n_{\text {bin }}=$ number of pieces of luggage already in bin, and

$n_{l}=$ number of pieces of luggage carried by passenger.

Fractional results for $t_{s l}$ are rounded to the next integer. It should be noted that all passengers in this simulation carry at least one piece of luggage.

The value of $n_{\text {bin }}$ refers to the corresponding half-row beneath the bin; passengers always put their luggage into the bin corresponding to their half-row. In reality, if the overhead bin becomes full, passengers may have to move to other rows to find a suitable location for their luggage. This aspect is not reproduced directly by the simulation; however, it should be noted that $t_{s l}$ becomes rather large for full bins. (The equation used by VLB was not available.)

\section{Seating Model}

The time passengers need to sit down depends on the number of obstructing passengers who are already seated. Those obstructing passengers have to get out of their row and then sit down again after the other passenger has been seated. The mathematical form (once more in simulation time steps) is

$t_{s}=t_{p}+2 * t_{p} * n_{s}=t_{p}\left(1+2 n_{s}\right)$

where

$t_{s}=$ total time for seating (simulation time steps);

$t_{p}=$ time used to get from seat into aisle or back (steps), $t_{p}=1.5$; and

$n_{s}=$ number of obstructing passengers already seated.

Results are also rounded to the nearest integer. 
VLB's formula was not available. Their text implies that they use $t_{s}=n_{s} t_{\text {out }}+\left(n_{s}+1\right) t_{\text {in }}$, with different times $t_{\text {out }}$ and $t_{\text {in }}$ for getting up and sitting down. Those times are triangular distributions; in simulation time steps, the values of the corner points are (1.25, 1.5, 1.75 ) (mean 1.5$)$ and $(2.5,3.75,12.5)$ (mean $\approx 5.9)$. Therefore, $t_{\text {out }}$ in this simulation is similar to theirs, but the $t_{\text {in }}$ here is considerably faster. This fact should be kept in mind since it will explain why their results have larger differences between conflict-rich and conflict-poor strategies than the current results do.

\section{Disturbances}

In this simulation different disturbances are introduced as follows:

- Early or late passengers, or both. If passengers are divided into boarding groups, it will often occur that some arrive late or early. The number of these passengers will increase with the number of boarding groups. With the ticket reader system, the boarding staff has the possibility to reject passengers who line up in a earlier boarding group. For travelers who arrive late, access is always granted. It will be seen how much the ratio of late- and early-arriving passengers will influence the quality of the boarding strategies.

- Aircraft dimensions. A boarding strategy should be robust under the use of different airplane layouts.

- Airplane occupancy level. Airplanes are not always full, and therefore boarding strategies should be efficient even with smaller occupancies. However, boarding with the same strategy but with fewer passengers will on average always be faster than when the plane is full. As long as the scheduled turn times (and therefore the flight schedule) are not adjusted to the expected demand, there is little need to test boarding strategies for reduced occupancy. Nevertheless, for completeness such results will be added. For reduced occupancy, four criteria influence which seat a passenger is assigned:

- To avoid balancing problems, the number of passengers sitting on the right side of the aisle should be about equal to the number of passengers sitting on the left side;

- For the same reason, the number of passengers sitting in the front area of the airplane should be equal to the number of passengers sitting in the back area;

- Window and aisle seats are assigned first; and

- Passenger seat preferences (e.g., exit row, seat near front of airplane for quicker exit) are not modeled by the simulation and will therefore not be taken in further account.

Technically, the simulation first assigns all window seats randomly, then all aisle seats randomly, and then all center seats randomly. That is, there are no preferences for any part of the airplane, but fluctuations generated by the randomness are accepted.

\section{Boarding Strategies}

In the first step the same boarding strategies as those of VLB are simulated under varying conditions. Later, improved boarding strategies are compared with the same nomenclature (these strategies are also shown in Figure 1).

- Block. To build boarding groups, the airplane is divided vertically (from back to front) into blocks.

- Half_block. The airplane is divided into boarding groups vertically and horizontally (right and left side of the aisle).
- "Block_des" means that the blocks are announced in descending order.

- "Block_X_alt_Y" means that there are $X$ blocks, which are announced in alternating order, skipping $Y$ blocks. For example, in a scheme with five blocks one could first call them in the sequence 5 , 3, 1, 4, 2, which would be called block_5_alt_1.

- Row. Every boarding group corresponds to a row. For an airplane with $R$ rows, block $\_R_{-} \ldots$ and row $\ldots$... are the same.

- Half_row. Every boarding group corresponds to the half of the row divided by the aisle.

- "Row_alt_Y" means once more that $Y$ rows are skipped.

- Letter. The letter of the seat indicates the place in a row; every boarding group corresponds to one or more letters or columns. When the airplane is boarded from the front, and the front is assumed to be on the bottom, letters ascend from the right to the left; "letter_ wintocorr" means sequence F, E, D, A, B, C; "letter_alt" means sequence F, A, E, B, D, C. No systematic differences between these two strategies are expected; "letter_outsidein" means sequence (F,A), $(\mathrm{E}, \mathrm{B}),(\mathrm{D}, \mathrm{C})$, where the parentheses indicate that the corresponding columns are boarded as one boarding group.

- Seat. The sequence of every single passenger is determined, and every boarding group consists of only one seat. The advantage of this procedure is that passengers can be lined up exactly; for example, one can, for letter $F$, have the passengers enter exactly in the right sequence, then for letter E, and so on; this is called seat_des_row_letter. It is intuitively clear that this is a good strategy. However, because the number of luggage pieces varies stochastically from one passenger to the next, it is not necessarily absolutely optimal for a given set of passengers with given amount of luggage. Nevertheless, the simulations confirm that this strategy has the best average performance. However, it is too complicated for real-world use.

To gain some more insight into these complex strategies, variations of seat strategies are tried. These complicated variations are shown graphically in Figure 1.

Both "letter" and "seat" strategies force row neighbors to enter the airplane at different times. This strategy may be undesirable when row neighbors know each other and want to travel as a group.

\section{Announcement Systems}

To control the sequence of boarding groups, an announcement system is needed. Typically, gate agents announce which boarding group is allowed to board. The passengers are often called in rows, for example, Rows 10 to 15 . Alternatively, boarding groups could be denoted by numbers on the boarding cards or by colors of the boarding cards and be announced or indicated by colored lamps. Another possibility is the use of numbered tickets and displays that indicate the current boarding number comparable with those used in banks, post offices, or supermarkets. An alternative to the display would be the use of numbered marks on the floor, at which people would have to line up before boarding. The last two systems are only applicable if passengers board through a finger dock.

\section{VALIDATION}

The same boarding strategies as those of VLB were modeled. To be consistent with their work, five replications of each strategy were 


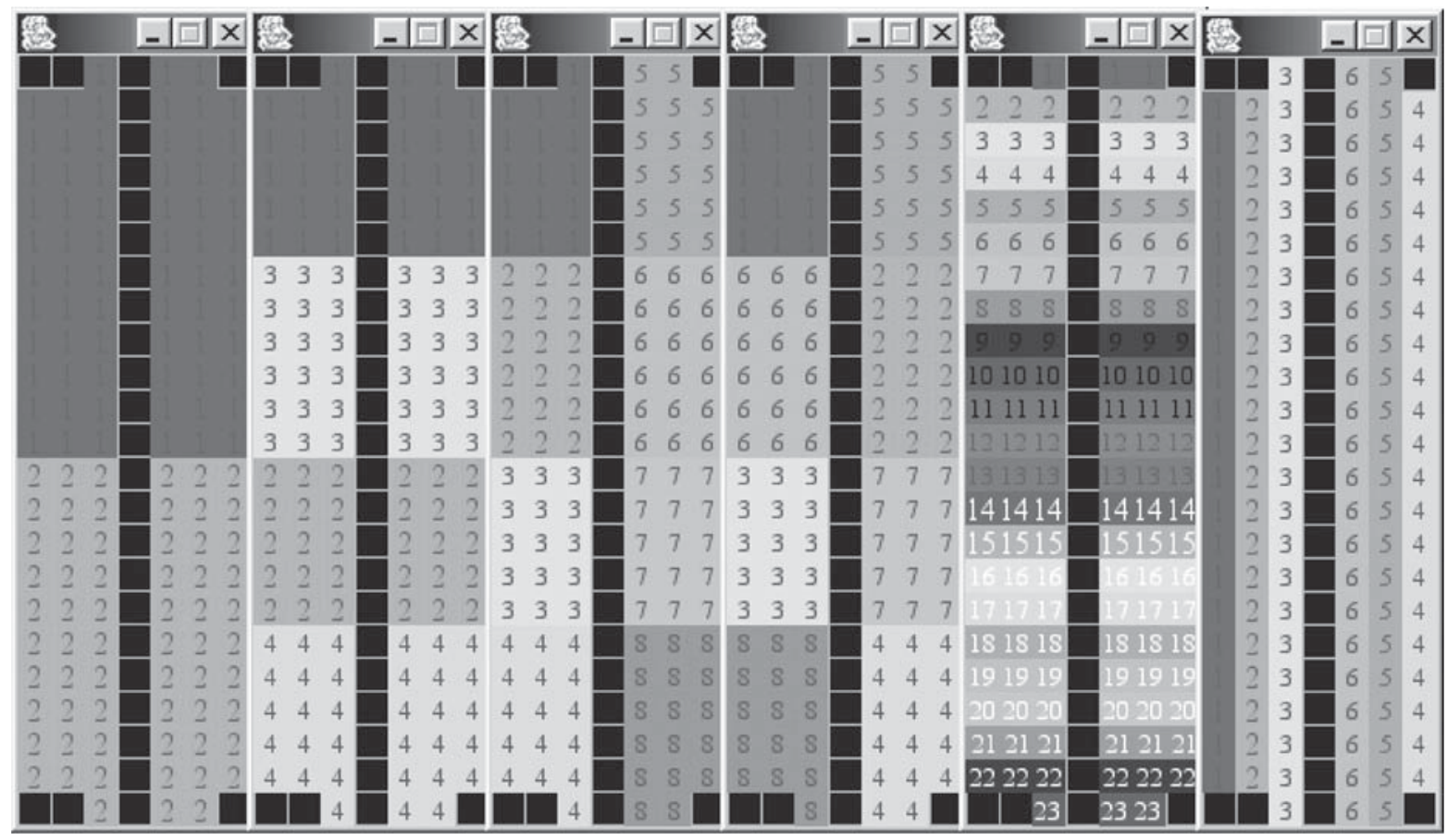

(a)

(b)

(c)

(d)

(e)

(f)

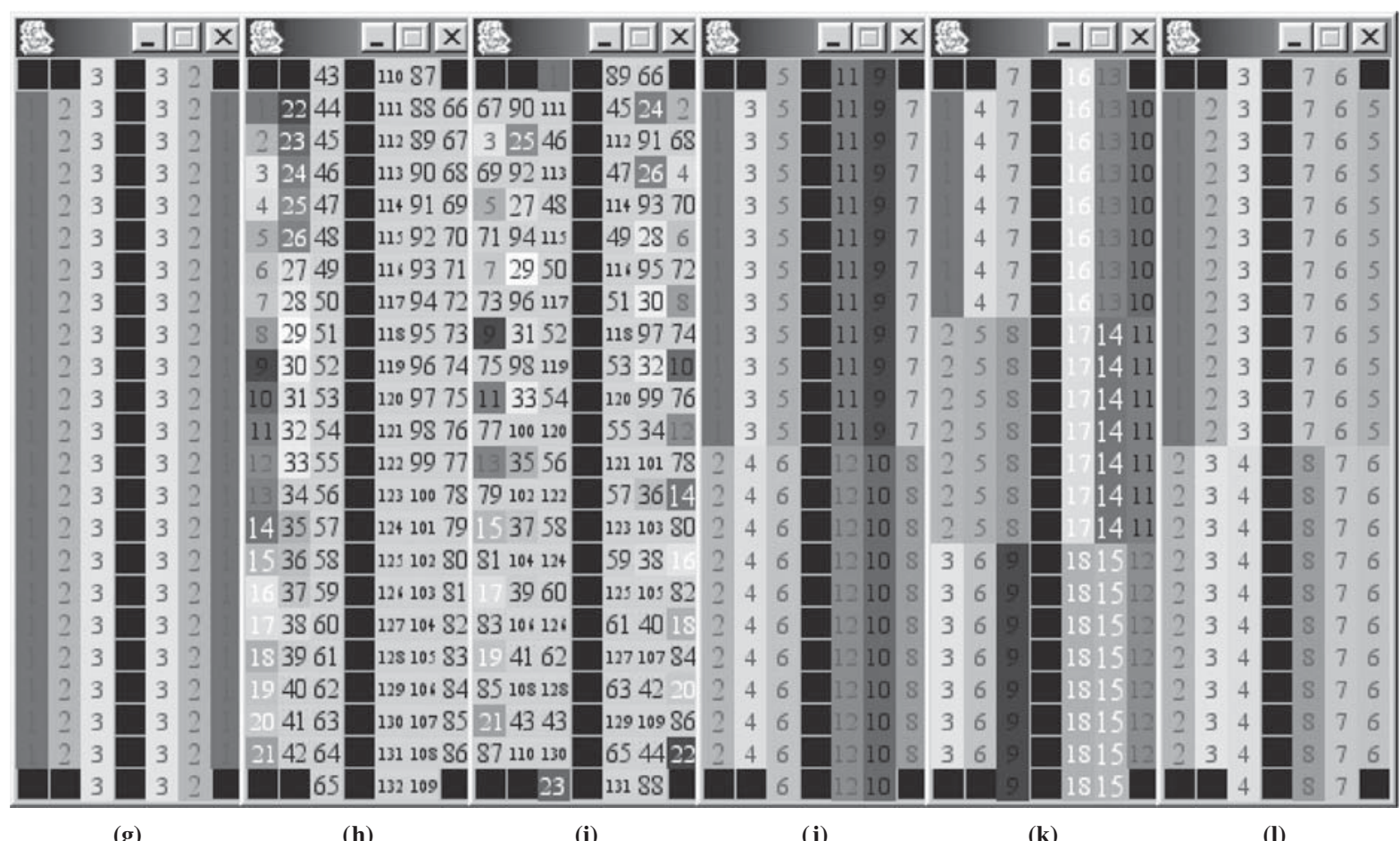

(g)

(h)

(i)

(j)

(k)

(I)

FIGURE 1 Graphical representation of boarding strategies: (a) block_2_des, (b) block_4_alt_1, (c) halfblock_4_des, $(d)$ halfblock_4_des_mix, $(e)$ halfrow_des, $(f)$ letter_wintocorr, $(g)$ letter_outsidein, $(h)$ seat_des_row_letter,

(i) seat_des_row_alt_letter, ( $j$ ) seatgr_2_des_row_letter, $(k)$ seatgr_3_des_row_letter, and (/) pyramid_2_des. 
performed and then averaged. The occupancy level of the airplane is $100 \%$, and all kinds of disturbances are ignored.

\section{Average Boarding Time}

For a comparison of the results for average boarding times, see Figure 2. Simulation time steps are multiplied with $2.4 \mathrm{~s} / \mathrm{step}$ as is plausible from the section on the passenger model.

The somewhat surprising result is that the much simpler simulation regenerates nearly exactly the performance profile of VLB. If one multiplies the results of Figure 2 by 1.25, these results would nearly completely coincide with the VLB results. In fact, the most important exception is the optimal strategy seat_des_row_letter, the advantage of which over the other strategies is less in the current simulations than in theirs. VLB were contacted about possible reasons for that difference but no reply was obtained. Yet, even without complete understanding of those differences, it is a strong indication for the robustness of these simulations that a simple reimplementation with a different technology leaves the relative strengths of the different strategies completely intact.

The general interpretation of Figure 2, consistent with VLB, is as follows:
- Block strategies (block_N_X) are most efficient with just one boarding group and become increasingly inefficient with more boarding groups. It should be noted that block_1_des is just plain random boarding.

- Filling the airplane from the back row by row is inefficient because there are always conflicts in the area where passengers are in the process of seating themselves, whereas no seating is done in other areas of the airplane. Row strategies become better when rows are skipped.

These foregoing two results already lead to an important conclusion: boarding by row from the back is inefficient because of localized conflicts. Making the blocks larger reduces those conflicts, and they are maximally removed when there is only one block, that is, random boarding. VLB had exactly the same result.

- The performance of left-right block strategies (i.e., first left, then right; halfblock_N_X) is slightly better than that of normal block strategies but not significantly better than random boarding.

- Combining left-right, by row, and alternating rows can be fairly efficient, as halfrow_alt_2 shows. Unfortunately, this strategy is rather complicated. VLB explain it thus: such strategies are efficient if the number of "jammed" people fits in between the "busy" rows. For

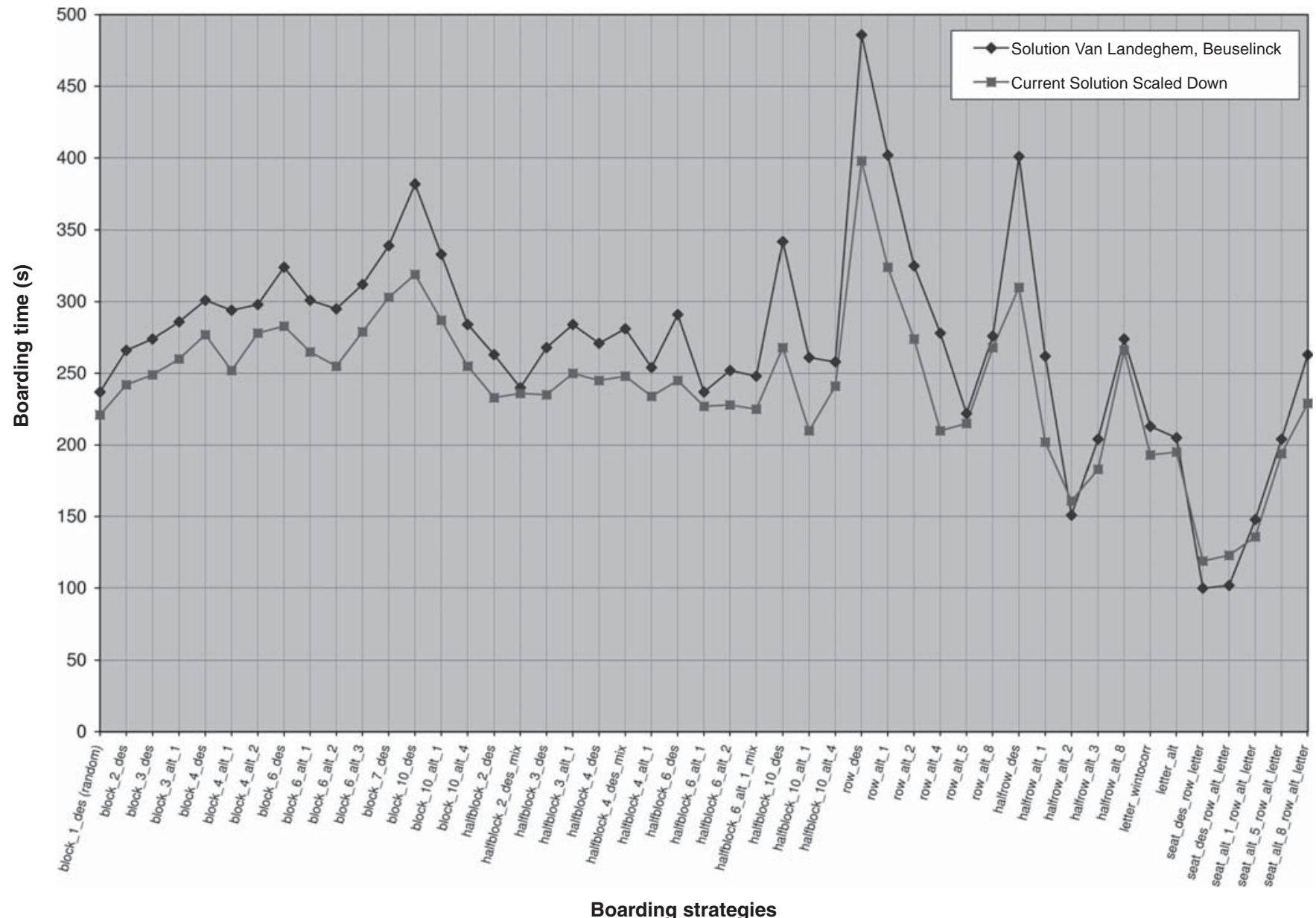

FIGURE 2 Comparison of average boarding times; conversion of simulation steps into seconds is based on 2.4 s/simulation step, as explained in text. 
example, if passengers board by half-rows, three people are busy with a half-row, using up the row itself plus two rows upstream. Therefore, those two rows need to be skipped in order to arrive at the next row that can be used efficiently. This process leads, with the airplane in this simulation, to alt_2 for efficient half-row strategies, and to alt_ 5 for efficient row strategies.

- Boarding from the window to the aisle (letter_wintocorr) is more efficient than random boarding.

- Completely determining the boarding sequence allows significant improvements, nearly halving the boarding time when compared with random boarding or block boarding. However, this strategy is even more complicated than halfrow_alt_2.

The overall result is that block boarding is even less efficient than random boarding (i.e., no system at all), and that within the considered strategies, there are no simple, good alternatives. This finding is consistent with VLB's results.

At first glance, it is not obvious why the block strategies fail. The graphical representation of the simulation reveals that many passengers are sitting in the same row as the passenger immediately ahead of them. Since that passenger ahead blocks the aisle while he or she is storing his or her luggage, the consequence is a backup. This effect increases with the number of blocks since there is a higher probability that passengers entering together will also be in similar rows. In contrast, alternating the sequence can help. In all cases, random boarding is more efficient and does not need an announcement system. From the point of view of boarding efficiency, there is no reason for using block strategies.

\section{Average Worst Case}

As discussed earlier, for airlines that are introducing new boarding strategies, not only the average boarding time is of interest but above all the possibility of long boarding times. To take this situation into consideration, boarding strategies will no longer be ranked according to the average boarding time but according to the average worst case of the boarding time measured in simulation time steps. The average worst case is calculated using the root-mean-square error (RMSE), as follows:

average worse case $=$ average boarding time $+3 *$ RMSE

where

$\mathrm{RMSE}=\sqrt{\frac{1}{n} * \sum_{i=1}^{n}\left(s_{i}-\bar{s}\right)^{2}}$

$s_{i}=$ boarding time of run $i$,

$\bar{s}=$ average of all boarding times for this strategy, and

$n=$ number of replications.

To justify the use of the RMSE, Figure 3 shows that the distribution of boarding times is symmetrical. This plot is representative for all other strategies examined. Although no further attempts have been made to justify that the underlying distribution is exactly Gaussian,

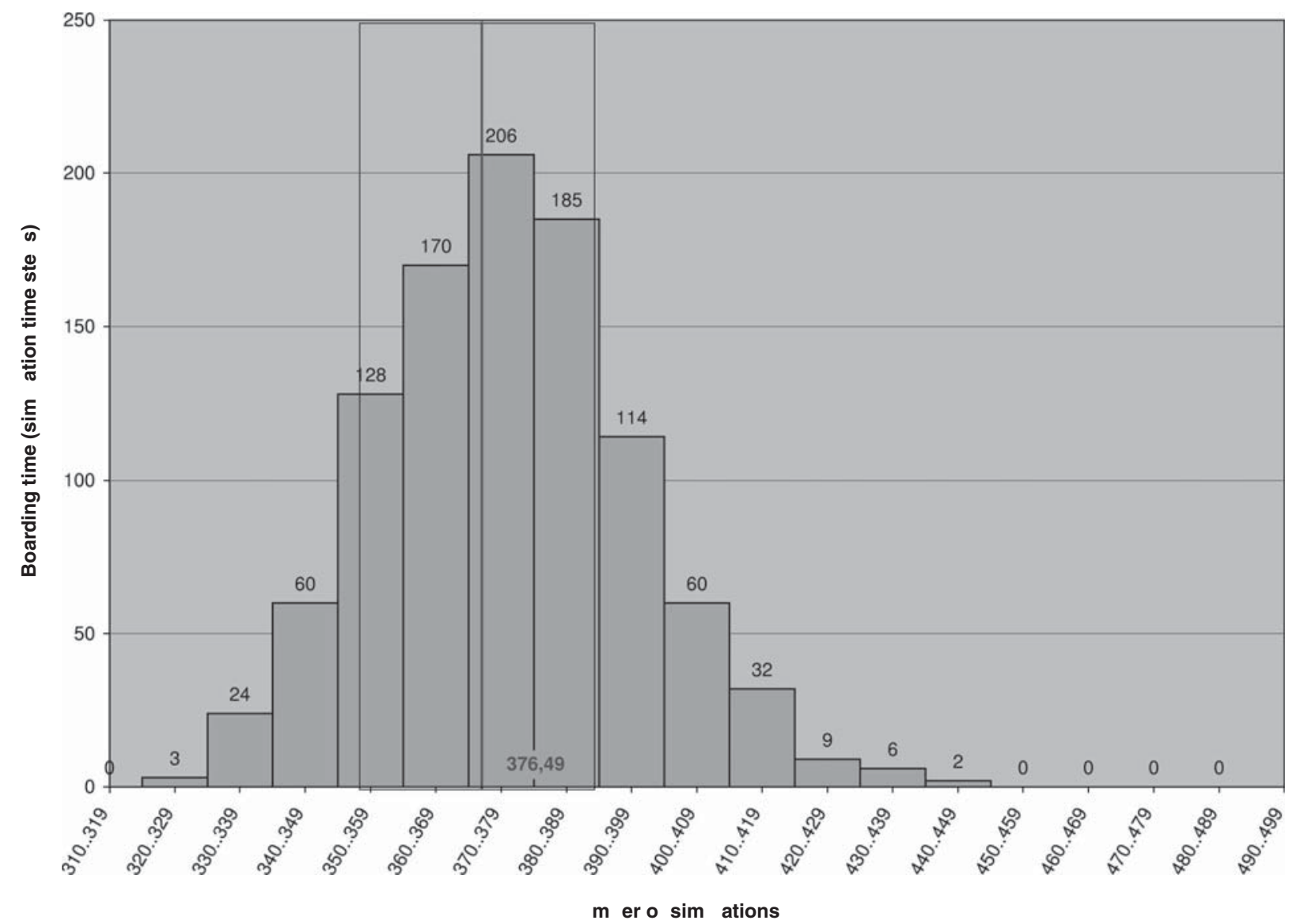

FIGURE 3 Distribution of seat_des_row_letter, 20\% late passengers. 
the plausible interpretation of this measure is that approximately 95\% of all boarding events are faster than the number found in this study.

Average worst performance is shown, together with average performance, in Figure 4. Boarding strategies that have good average performance also have good average worst-case performance. Importantly, the absolute difference between average standard and average bad performance increases with increasing average boarding time. That is, strategies that are already bad have even stronger fluctuations to the worse. Nevertheless, the ranking of the strategies according to worst-case performance is similar to the ranking of the strategies according to average performance. VLB found similar results.

\section{ROBUSTNESS OF BOARDING STRATEGIES UNDER DISTURBANCES}

An important aspect of real-world boarding is that passengers often do not follow their boarding groups. Passengers attempting to board early can be stopped by the airline staff, but at the expense of bad customer relations. Nothing reasonable can be done about late passengers. The important question here is how much early and late boarders affect the efficiency of the strategies. Two questions seem of particular importance:

- What is the advantage or disadvantage if staff let early boarders slip through?

- What is the average worst performance of a strategy? A strategy with a good average performance but frequent outliers may be less desirable for an airline than a strategy that is worse in the average but more reliable.

In contrast to the modeling in the section on validation, 50 replications of the runs were used to achieve more accurate results.

\section{Effect of Early and Late Passengers}

To examine the effect of early and late passengers on the average worst case, 50 replications were performed on every strategy using a full aircraft. The case in which a given percentage of the passengers enters late and the case in which they arrive early as well as late were simulated. Results showed that there is no significant difference between the two possibilities. In other words, if $20 \%$ of the passengers

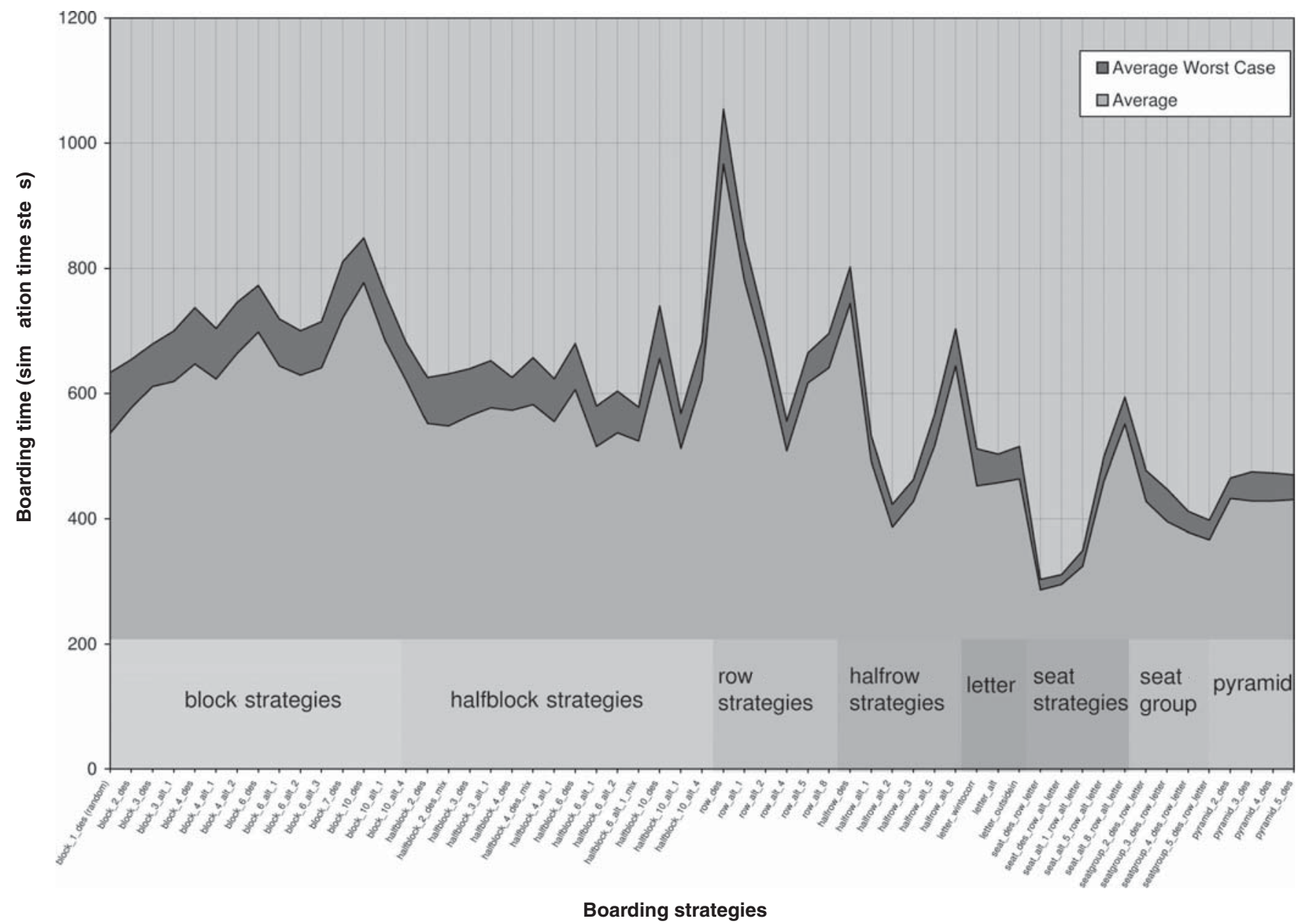

FIGURE 4 Average boarding times versus average worst-case boarding times. 
board at the wrong time, half of them early and half of them late, the effect of the disturbances can be reduced to $10 \%$ if all early boarding attempts are rejected.

In Figure $5 a$ curves are plotted for the average worst case with 20\%, $40 \%$, and $80 \%$ of passengers arriving late. When increasing fractions of passengers arrive late, the boarding time approaches the value of the block_1_des strategy, since that strategy just means that all passengers enter randomly.

The simulations show that with disturbances, block strategies continue to perform worse than plain random boarding. More important, the more passengers do not follow the boarding groups, the better the block strategies become. The effect becomes more pronounced with more boarding blocks. In some sense, this result is clear since it was already established that random boarding performs better than boarding by block, and so, clearly, introducing randomness will pull the block boarding strategies toward random boarding performance. In another sense, however, the result is quite troubling, because it implies that a passenger not obeying the airline boarding announcement in fact improves boarding efficiency.

Since this result goes against conventional wisdom, an expansion on this point is in order. Boarding back to front essentially means that there is a lot of conflict-causing loading activity in the current boarding block, whereas there is no loading in other parts of the airplane. In this situation, passengers boarding at times when they are not called means that they will be loading in areas of the airplane with little current activity, thus increasing the amount of loading that can occur at the same time.

Descending half-block strategies are stable for passenger conflicts but do not improve the efficiency compared with the random strategy. The average worst-case boarding time will increase with the number of half-blocks for the same reason as for block strategies. Alternating half-block strategies seem to be stable also; halfblock_ 6_alt_1 and halfblock_10_alt_1 obtain good performance. These strategies need twice as many boarding groups as do comparable block strategies. Half-block strategies are also not recommended, but if they are used anyway, alternating variants should be preferred.

The highest (= worst) peak in Figure $5 a$ belongs to the row strategy because every seat interference will interrupt the boarding of the actual group. Alternating row strategies can improve this situation once more.

The descending half-row strategy results in bad performance. Alternating the half-rows helps greatly, but the strategies seem to become very unstable if passengers do not arrive in time. The probability for passengers arriving late can be expected to be quite high as a consequence of small boarding groups. Alternating half-row strategies are only recommended in combination with a reliable announcement system.

Letter strategies show an acceptable stability and are easy to introduce in practice since they need small numbers of boarding groups. Using letter strategies is recommended.

Seat strategies determine the sequence of boarding passengers at the individual level. Every boarding group has as it were only one member. In practice it will be costly to introduce such a system, and in addition it needs to be very reliable because of great lack of robustness in the case of passenger disturbances.

\section{Free Seat Choice}

Some airlines do not offer assigned seats at all; passengers choose their favorite seat once they are inside the airplane. Since the number of tickets sold is limited to the capacity of the plane, no booking system is necessary. This kind of boarding puts travelers under pressure; it is therefore unsuitable for business- or first-class passengers.

Models of free seat choice are more difficult to simulate than models with fixed seats since for the former a model of human behavior needs to be included. For these simulations, the following assumptions were made:

- Window seats and seats near the aisle are the passengers' favorites;

- Free rows will be preferred;

- Before sitting down, the passenger will ensure that there is no better place in the next few rows;

- If passengers are queuing, it is possible that they will lose patience and accept a more unsuitable seat than the one they expected;

- If a passenger arrives at the last row with free seats, he or she will sit down there; and

- Passengers will not change their walking direction to find a seat.

It is hard to predict the exact behavior of passengers, and other assumptions will probably lead to different results. Nevertheless, a good impression of how this strategy will work can be obtained. The simulation shows that at the beginning people board the plane quickly. Later the strategy gets very inefficient because of increasing seat interferences (middle seats are occupied last) (curve labeled "passengerSW" in Figure 6). Free seating should not be used if rapid loading of fully booked airplanes is the objective. However, if low administrative overhead is needed and airplanes are usually not full, then it is a viable alternative.

\section{Improved Boarding Strategies}

An attempt is made to modify the best case (seat_des_row_letter) to decrease the number of boarding groups while retaining as much of the good performance as possible. As will be seen, strategies exist that are reasonably simple but still fast and robust.

- Seat-group strategy. The airplane is filled corresponding to the seat_des_row_letter strategy, but instead of single seats, groups of seats are used. The seating is divided horizontally into a specified number of groups and vertically by columns (letters). The boarding groups are called in descending order from back to front and from the outside to the inside. For an airplane with $R$ rows, seatgroup_ R_des_row_letter and seat_des_row_letter are the same.

- Pyramid strategy. The number of boarding groups can be further reduced while some of the good performance of the seat-group strategies is retained by merging passenger groups from the seat-group strategy diagonally. We will call this strategy “pyramid_des” since the passengers board the craft in a pyramidal shape. This strategy is a combination of outside-in and back-to-front: the window seats at the back of the plane are boarded first (Group 1); then the window seats in the middle part of the plane and the middle seats at the back of the plane are boarded (Group 2); then the window seats at the front of the plane, the middle seats in the middle of the plane, and the aisle seats at the back of the plane (Group 3); and so on. Supposedly, American Airlines is already using this strategy.

The right-hand side of Figure $5 a$ shows the two improved strategies just described. They achieve very fast boarding. Pyramidal boarding needs fewer boarding groups than seat-group boarding but is slightly slower. Both strategies are recommended. 


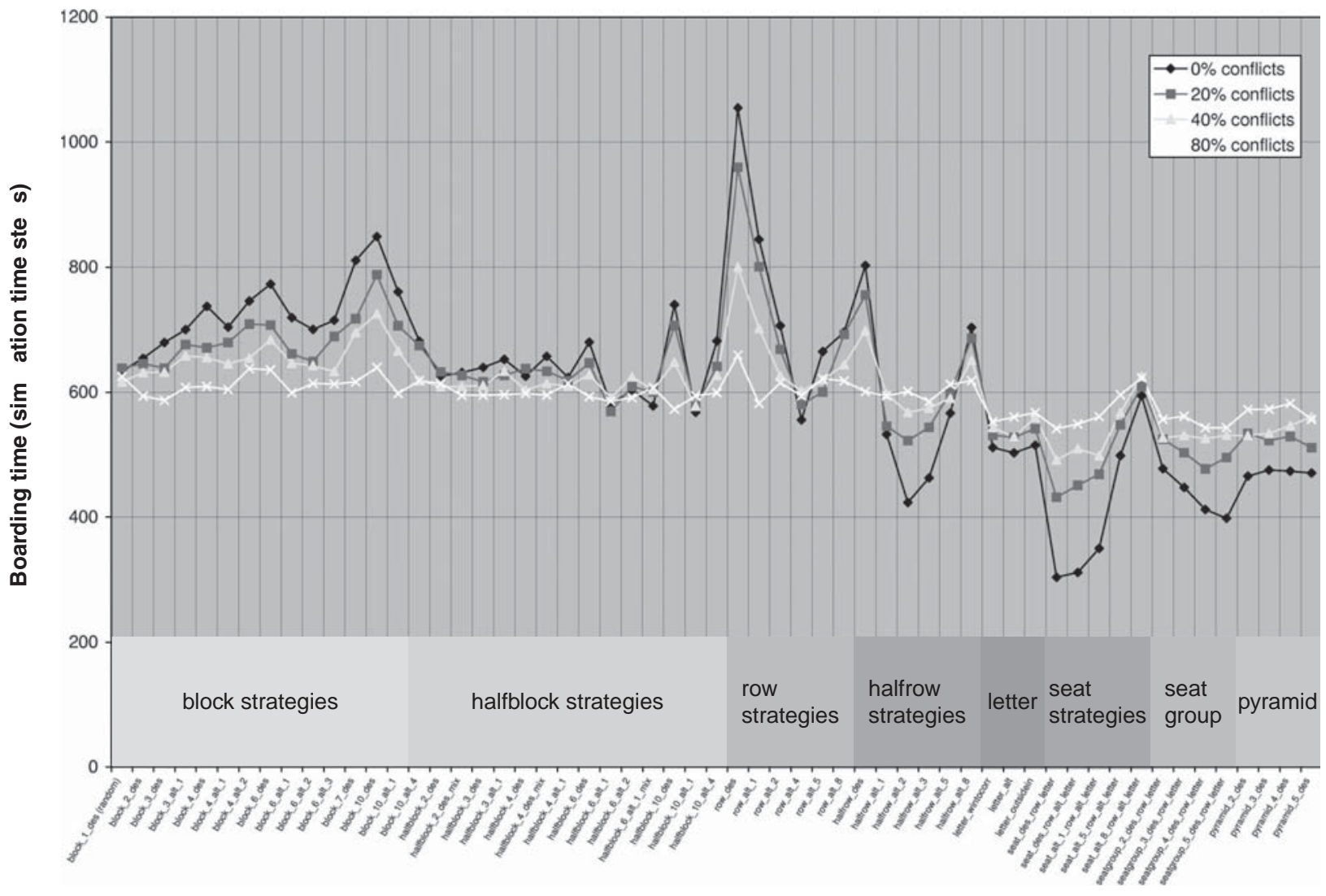

Boarding strategies

(a)

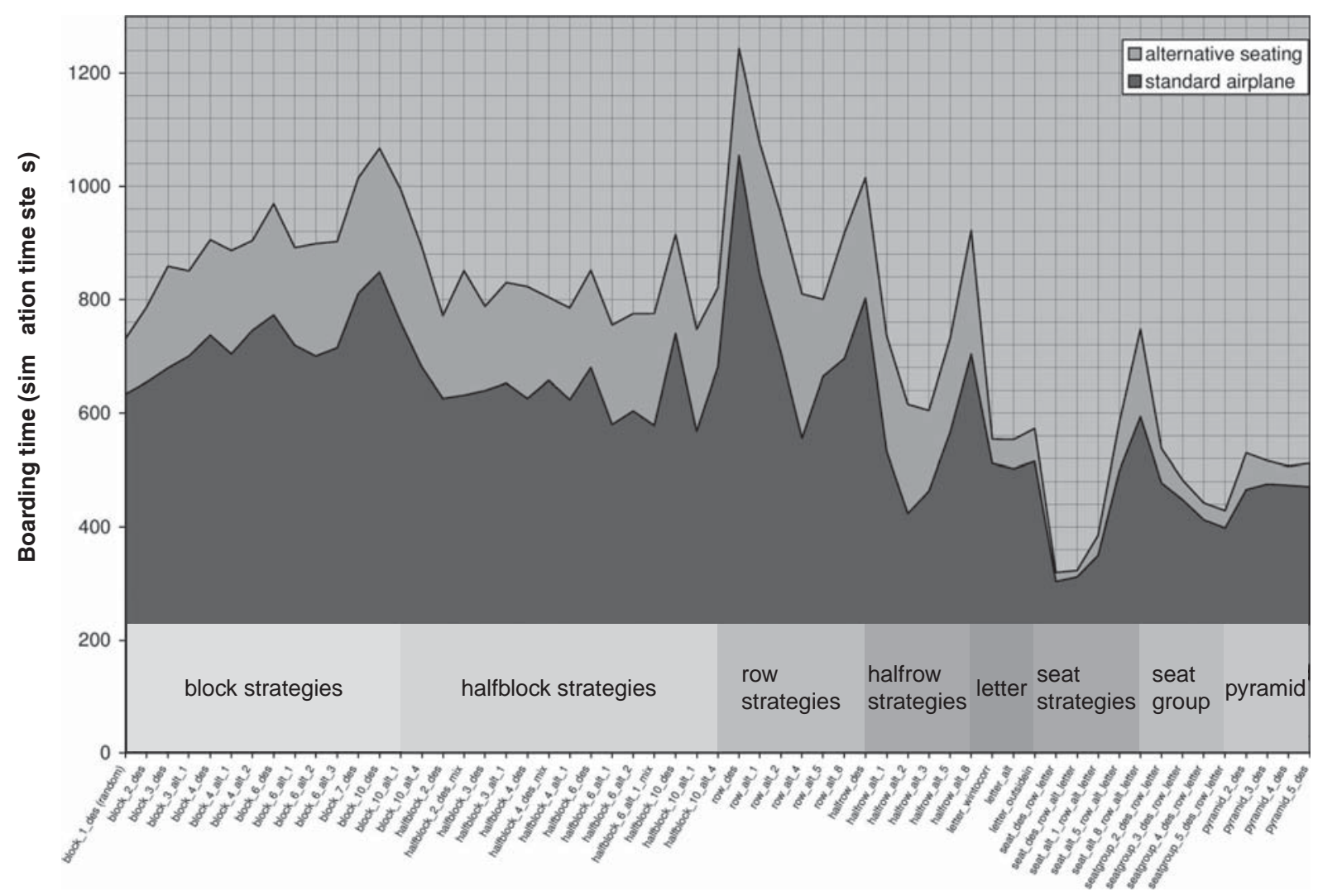

Boarding strategies

(b)

FIGURE 5 Effect of disturbances (passengers boarding late): (a) average worst-case boarding time with 0, 20\%, 40\%, and 80\% of all passengers entering late, and $(b)$ average worst case for "standard" airplane and for a different layout of same number of seats with eight instead of six seats per row. 


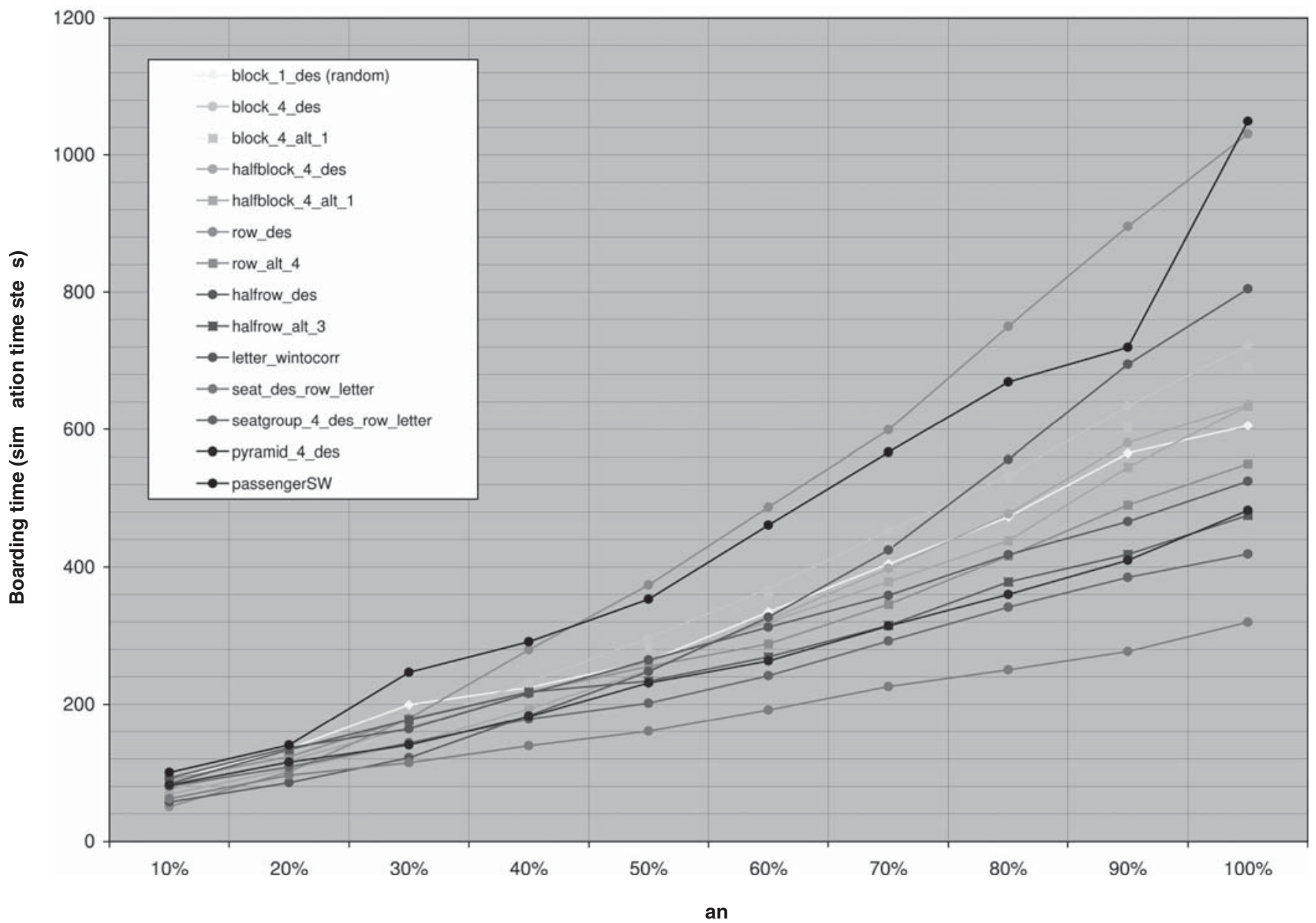

FIGURE 6 Average worst case of chosen strategies under different occupancies.

One should, however, note that all efficient strategies have a tendency to separate row neighbors from each other. This arrangement may not be desired by passengers traveling together. However, conflicts to a large extent stem from passengers entering a row in the wrong sequence. Since passengers traveling together would not be expected to enter in that manner, there is a good chance that leaving row neighbors together will not make the boarding system inefficient even if it is inconsistent with the boarding group. Such a strategy could be tested in future work.

\section{SENSITIVITY}

\section{Effects of Aircraft Dimensions}

Boarding strategies are always ranked with reference to the standard airplane dimensions. Some strategies are expected to be independent of the aircraft model.

The same simulations are performed on a second aircraft model with the same number of seats but with eight instead of six passengers in every row. Figure $5 b$ shows the average worst cases for the standard and the modified airplane model. As one can see, the boarding takes more time overall, but some strategies behave particularly badly, for example, row_alt_4 or halfrow_alt_2. In contrast, seat strategies are more robust, as are letter, seat-group, and pyramidal strategies. The other strategies show great instability.

The size of the luggage bins remains unchanged, which in the current model formulation means that the fourth passenger in a row is faced with strongly increased luggage-storing times. Nevertheless, the fact that the fastest strategies are nearly unchanged between the two airplane layouts indicates that luggage storage is only a small part of the boarding time.

The conclusion is that if a robust boarding strategy is intended to be used for different seat layouts, only letter, seat, seat-group, and pyramidal strategies are recommended.

\section{Effects of Aircraft Occupancy}

To find out how the efficiency of strategies depends on aircraft occupancy, a representative strategy was chosen for every group. These strategies were evaluated under different occupancies between $10 \%$ and $100 \%$. The plots of the results are more or less parallel. If the airplane is loaded more than $50 \%$, the scoring remains almost the same. Some strategies seem to work slightly more efficiently than others if the occupancy remains under $40 \%$. This behavior will not 
influence the final results because in these cases the time required for passengers to board is short and will therefore affect the turn time very little. However, average occupancies can expected to be higher than $50 \%$.

\section{Aspects of Luggage Loading}

A currently much-debated issue is how far the restriction of carryon luggage will reduce boarding times. In general, just restricting the amount of carry-on luggage will accelerate the boarding process, since the average luggage storing times according to Equation 1 will be reduced. The issue gets more complicated when there are correlations, for example, privileged passengers with more carry-on luggage concentrated in the front of the airplane. Such considerations were outside the scope of this study. However, the microscopic simulation approach would easily allow such aspects to be added and to be evaluated in a systematic way. Since this evaluation involves correlations between seating and carry-on policy, it should probably be done in collaboration with an actual airline.

\section{SUMMARY}

The following results may be summarized:

- Using a model for airplane boarding that is only roughly based on previous work reproduces the results surprisingly well.

- The often-used block strategies are inefficient since they prolong the passenger boarding process as compared with random boarding. In fact, passengers ignoring the boarding announcements improve the performance of those strategies.

- Half-block, row, and half-row strategies are not recommended; the benefit is too small compared with the large number of boarding groups. Some of the half-row strategies show good performance, but that good performance is highly dependent on changes in the skip amount (number of skipped rows between boarding groups).
- Boarding-by-column (letter) strategies are recommended. They are less efficient than some of the half-row and the explicit seat boarding strategies but more efficient than the block strategies. Also, the result is more robust than that for the good half-row strategies in the sense that it does not depend on implementation details such as the exact number of skipped rows. Finally, the number of boarding groups is relatively small.

- The best choice is the seat-group strategy. It provides excellent efficiency and good stability combined with a relatively small number of boarding groups.

- It is possible to combine some of the seat groups in a diagonal pattern. This procedure leads to a small number of boarding groups, with a performance that is still better than that of random boarding.

- The good strategies are reliably good even for changes in aircraft layout and for occupancies less than $100 \%$.

\section{REFERENCES}

1. Van Landeghem, H., and A. Beuselinck. Reducing Passenger Boarding Time in Airplanes: A Simulation-Based Approach. European Journal of Operational Research, Vol. 142, 2002, pp. 294-308.

2. Van den Briel, M. H. L., J. R. Villalobos, and G. L. Hogg. The Aircraft Boarding Problem. Proc., 12th Annual Industrial Engineering Research Conference (IERC-2003), Portland, Ore., 2003, No. 2,153.

3. Marelli, S., G. Mattocks, and R. Merry. Racing the Clock: The Role of Computer Simulation in Reducing Airplane Turn Time. Aero Magazine, No. 1, 1998, p. 10. www.boeing.com/commercial/aeromagazine. Accessed Sept. 2003.

4. Kirchner, A., H. Klüpfel, K. Nishinari, A. Schadschneider, and M. Schreckenberg. Simulation of Competitive Egress Behavior: Comparison with Aircraft Evacuation Data. Physica, Vol. 324A, 2003, pp. 689-697.

5. Bachmat, E., D. Berend, L. Sapir, and S. Skiena. Airplane Boarding, Disk Scheduling, and Space-Time Geometry. Proc., Algorithmic Applications in Management 2005, 2005, pp. 192-202. 